\title{
Seeing is Educating: The Case for Electron-Microscopy Videos in the Classroom
}

\author{
Alyssa J. McKenna and David J. Flannigan* \\ Department of Chemical Engineering and Materials Science, University of Minnesota, 421 Washington Avenue SE, Minneapolis, \\ MN 55455
}

*flan0076@umn.edu

Owing to the gap between the spatial resolution limit of the human eye and the nanometer scales at which the properties of solids originate, microscopy has played a key role in our understanding of engineering materials. Feynman, in his unique and highly coherent way, spelled this out and, in a sense, challenged experimentalists to push the limits of electron microscopy such that imaging of atoms and their movements could be achieved [1,2]. Since 1960, advances in electron microscopy that perhaps even he could not have foreseen have been realized, resulting in the redefinition of spatial and temporal limits. We must do more to bring video images of atomic movements to the next generation of scientists and engineers.

While they are often powerful and inspiring, static images of atoms, atomic arrangements, and structural morphology are inadequate to fully illustrate processes that occur in materials. Ideally, one would watch events unfold on the spatial scales pertinent to the phenomenon of interest. In electron microscopy, such capabilities have produced a number of new discoveries $[3,4]$. Perhaps a bit lost in the clamor of discoveries and new observations is a potential second benefit, which is the dramatic impact video clips of dynamic processes in materials could have on budding scientists and engineers if extended to the classroom. We argue that serious thought should be given to incorporating such videos into the early stages of undergraduate education.

Education research indicates that classroom demonstrations and active-learning environments engage students and aid in solidifying their comprehension of the underlying scientific concepts $[5,6]$. We feel an ideal way to facilitate this would be to have an open-access database comprised of in situ electron microscopy videos that directly illustrate-in a dramatic, visual fashion-certain fundamental concepts of materials science (for example, elastic and plastic deformation, dislocation motion, phase transitions, nucleation and growth, etc.). Instructors could access and use these videos in their classrooms at the appropriate point in the unit, with the intent being to engage and excite the students' interest in the topics under discussion.

An approach taken by Flannigan in teaching introductory courses in science and engineering at the University of Minnesota has been to incorporate videos from his own research, illustrating fast and ultrafast materials phenomena into his lectures. Such videos, even in lectures of $150+$ students, have proven extremely fruitful in engaging the large class. For example, in one lecture, the elastic deformation of materials was discussed and approached from a potential energy perspective by posing the following question: "How can we determine elastic properties such as Young's modulus from a single, nanoscale crystal?" Next, a video was shown of nanosecond oscillations of a crystal visualized with state-of-the-art electron-microscopy techniques [7], and the students discussed how to deduce elastic properties from such data. That lecture is often one that students distinctly remember throughout the semester (and hopefully through the remainder of their undergraduate studies). In talking with them about such experiments, it is clear that a deep impression was made.

The enthusiasm expressed by students for the visualization of materials phenomena in this way suggests there are excellent opportunities for education and outreach using an open-access video database linked to specific textbook topics. For example, one might hold laboratory tours or summer schools for students wherein in situ atomic-scale measurements are performed. Laboratory modules on dynamic imaging could be incorporated into graduate-level courses on electron microscopy. Students in solid-state physics courses or kinetics courses could be coupled with the electron microscopy students to form a collaborative group of researchers for the semester. The possibilities are numerous and exciting.

To bring this excitement to our classrooms, we need an edited group of videos that can be easily accessed within lectures on particular materials-related topics. Some people might say we could just go to YouTube and pull down the relevant video clips, but these videos are unedited and not grouped in a fashion suitable for easy access by topic. What is needed is funding from a scientific society or university to create an open-access platform to mount these video clips. Furthermore, this project should have an editor to organize and update this valuable repository. A model for such an activity already exists: the CELL project sponsored by the American Society for Cell 
Biology: The Cell - An Image Library ${ }^{\mathrm{TM}}$ (http://www.cellimagelibrary.org/). Is there anyone in our community who can make this happen?

\section{Acknowledgments}

We acknowledge support by $3 \mathrm{M}$ in the form of a Nontenured Faculty Award under Award Number 13673369 and by the Donors of the American Chemical Society Petroleum Research Fund under Award Number 53116-DNI7. AJM is supported by the National Science Foundation Graduate Research Fellowship Program under Grant No. DGE-1348264. Any opinions, findings, and conclusions or recommendations expressed in this material are those of the authors and do not necessarily reflect the views of the National Science Foundation. Finally, we thank Charles Lyman for careful editing of the piece.

\section{References}

[1] RP Feynman, Eng Sci 23 (1960) 22-36.

[2] SJ Pennycook, MRS Bull 40 (2015) 71-78.

[3] H Zheng et al., MRS Bull 40 (2015) 12-18.

[4] DA Plemmons et al., Chem Mater 27 (2015) 3178-92.

[5] MC Buncick et al., Int J Sci Ed 23 (2001) 1237-55.

[6] M Prince, J Eng Ed 93 (2004) 223-31.

[7] DJ Flannigan and O Lourie, Microsc Anal 27 (2013)

$$
\text { S5-S8. }
$$

\section{Do You Know Your SEM X-ray Analysis Accuracy?}

- Most SEMs with X-ray analyzers in use today incorporate energy dispersive systems.

- Standardless analysis, the most often used method, produces results very quickly, but do you know how accurate it is (hint- this likely varies with different sample compositions)?

- One way to measure accuracy is to analyze calibration standards as unknown samples.

At Geller Micro̊̊nalytical Laboratory, we offer some 295 calibration standards that include pure elements, compounds, minerals, alloys and NIST standards all properly prepared for immediate use. Please visit our web site for 1 listings and prices then feel free to call us for advice.

We also manufacture and certify stage micrometers and magnification standards under ISO-17025 accreditation.

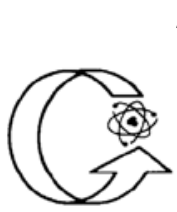

ISO-9001 certified for 16 vears!

GELLER MICROANALYTICAL LABORATORY, Inc.

426e BOSTON ST., TOPSFIELD, MA 01983-1216 TEL: 978 887-7000 FAX: 978-887-6671 www.GellerMicro.com

\section{Expand your Knowledge of Microscopy with} MSA Membership!

Whether your primary focus is in optical, electron or scanning probe microscopy, the biological or the physical sciences, MSA takes your knowledge to the next level!

Members Receive:

- A personal subscription to MSA's official journal, Microscopy and Microanalysis, and MSA's popular bi-monthly magazine, Microscopy Today.

- Peer Networking through the Society's Focused Interest Groups and Local Affiliated Societies.

- Plus discounts on books, journals and other educational materials.

- MSA Awards Programs, Scholarships, Speaker Opportunities, and much more!

For more information: visit www.microscopy.org or call 1-800-538-3672 